\title{
PSYCHOLOGICAL CONSEQUENCES OF OVER-MEDICALIZATION IN A PEDIATRIC PATIENT WITH FUNCTIONAL GASTROINTESTINAL DISORDER: A CASE REPORT
}

\author{
Consuelo Basile ${ }^{1}$, Federica Gigliotti ${ }^{1}$, Michela Colaiori ${ }^{1} \&$ Ignazio Ardizzone $^{2}$ \\ ${ }^{1}$ Multispecialty Department Child Neuropsychiatry, Department of Human Neurosciences, \\ Sapienza University of Rome, Rome, Italy \\ ${ }^{2}$ Multispecialty Department of Child Neuropsychiatry, Policlinico Umberto I Hospital, Rome, Italy
}

received: 20.11.2020;

revised: 14.2.2021;

accepted: 1.3.2021

$* * * * *$

\section{INTRODUCTION}

The prevalence of Functional Disorders (FD) in pediatric population is unknow; some studies show they could constitute the $25-50 \%$ of all visits in pediatric units (Malas et al. 2017); for others, one out of three access could be linked to somatoform symptoms (Bujoreanu et al. 2014). Most cases of FD show neurological or gastrointestinal symptoms (Malas et al. 2017). ROMA IV criteria are used for the diagnosis of Functional Gastrointestinal Disorders (FGD), those can coexist with other diseases (Hyams et al. 2016) and psychiatric disorders, especially anxiety and mood disorders (Bujoreanu et al. 2014). Doctors treating these conditions, may feel frustrated by not identifying specific causes; in addition, the patient and his family may feel underestimated, their compliance to treatment might be compromised (Malas et al. 2017).

\section{CASE REPORT}

This 8-year-old boy has been treated for six months by pediatric gastroenterology for recurrent abdominal distention with pain.

He suffered for allergic asthma and he underwent surgery for inguinal hernia. Physical examination revealed soft systolic murmur, distended but treatable abdomen and generalized joint hypermobility. Auxological parameters were normal.

Systemic infections, electrolytic imbalances, thyroid disorders, inflammatory bowel disease, celiac disease and other autoimmune disorders, malabsorption were excluded during hospitalization (see figure 1).

Abdominal ultrasound and Magnetic Resonance Imaging showed abdominal distension from jejune to the rectum, endoluminal air-fluid levels without mechanic obstruction. The main causes of chronic intestinal pseudo-obstruction were excluded (see figure 1). He started veal-free, egg-free, poultry and milk-free diet, in addition to treatment with antibiotic, prokinetic and antispasmodic drugs for several months without benefits.

During last medical checks, he presented tics (eyes rotation), loss of appetite and regressive behavior, therefore he was referred to our pediatric neuropsychiatry department for evaluation. Further investigation revealed that the mother was suffering from depression and anxiety; moreover, patient started to feel bad when his mother resumed work. Lastly, the patient had reduced his scholar attendance until he stopped it.

Our assessment showed he had normal intelligence with discrepancy among indexes, he failed in auditory and visual attention test, but his planning skill was preserved (see table 1).

No evidence of specific learning disabilities was identified. In addition, the patient manifested difficulties of separation from his mother, deficit in sustained attention and obsessive thoughts.

$\mathrm{He}$ was diagnosed with somatic symptom disorder and separation anxiety; he started pharmacological treatment with sertraline and psychodynamic psychotherapy plus family therapy (once a week), but sessions were discontinued due to abdominal disease relapse.

$\mathrm{He}$ was hospitalized again and the causes of intestinal dyskinesia were excluded (see figure 1). The measurement of abdominal circumference differed from morning to evening (from 2 to 5 centimeters). Evidences suggested a FD, hence a diagnosis of aerophagia was made and he was treated with rectal catheter to evacuate air.

A psychotherapy journey was initiated but he presented relapses of anxiety symptoms, he refused to continue the therapy without his mother; he had regression in autonomies (like showering himself) and he had oppositional behavior at his father and school refusal.

\section{DISCUSSION}

In the case is possible to identify some characteristics typical of FD. Anxiety and mood disorders in family members show genetic predisposition for psychiatry diseases (Malas et al. 2017, Bujoreanu et al. 2014, Sitholey \& Agarwal 2008); changes in family structure such as resumption of maternal employment (Malas et al. 2017, Bujoreanu et al 2014) is another frequent feature. Ultimately, protracted school absences are a common problem that can be considered a marker of social impairment (Bujoreanu et al 2014, Cozzi \& Barbi 2020). 
Table 1. Neuropsychological and psychodiagnostic assessment

Wechsler Intelligence Scale For Children-IV (WISC-IV)

$\begin{array}{lr}\text { Verbal Comprehension Index (VCI) } & 120 \mathrm{WP} \\ \text { Perceptual Reasoning Index (PRI) } & 100 \mathrm{WP} \\ \text { Intelligent quotient (IQ) } & 95 \mathrm{WP}\end{array}$

Working Memory Index (WMI) 85 WP

Processing Speed Index (PSI) $68 \mathrm{WP}^{*}$

A Developmental Neuropsychological Assessment (NEPSY II) Attention and executive functions

\begin{tabular}{|c|c|c|}
\hline \multicolumn{2}{|c|}{ Visual attention (AA) } & $4 \mathrm{SP} *$ \\
\hline \multicolumn{2}{|c|}{ Design Fluency (DF) } & $6 \mathrm{SP}$ \\
\hline Inibition A & TIME: $10 \mathrm{SP}$ & COMBINA \\
\hline Inibition B & TIME: 7 SP & COMBINA \\
\hline Inibition $\mathrm{C}$ & TIME: 5 SP & COMBINA \\
\hline \multicolumn{2}{|c|}{ Animal Sorting (AS) } & $10 \mathrm{SP}$ \\
\hline \multicolumn{2}{|c|}{ Auditory attention (AA) } & $6-10 \% \mathrm{P}^{*}$ \\
\hline \multicolumn{2}{|c|}{ Response set (RS) } & $6-10 \% \mathrm{P}^{*}$ \\
\hline
\end{tabular}

A Developmental Neuropsychological Assessment (NEPSY II) Social perception

Affect Recognition

$14 \mathrm{SP}$

Theory of Mind

$7 \mathrm{SP}$

Tower of London test (ToL)

Total score

$38 \mathrm{Ts}$

The bells Test

Time measure

$-1.6 \mathrm{Zs}$

Accuracy measure

$-1.54 \mathrm{Zs}$

Kiddie-Sads-Present and Lifetime Version- DMS 5 (K-SADS-PL-5)

Self-report: Tic Disorder (C), ADHD (C); OCD (B), EBD (B) Parents-report: GAD (B), ADHD (B)

Child Behavior Checklist 6-18 (CBCL)

Syndrome Scale Score Anxious/Depressed C

Withdrawn/Depressed B

Somatic Complaints C

DSM-Oriented Scales

Affective Problems C

Social Problems B

Anxiety Problems C

Somatic Problems B

Multidimensional Anxiety Scale for Children (MASC)

\begin{tabular}{|c|c|c|c|c|}
\hline \multirow[t]{3}{*}{ Physical Symptoms } & Tense/Restless & $51 \mathrm{Ts}$ & \multirow[t]{3}{*}{ Separation/panic } & \multirow[t]{3}{*}{$64 \mathrm{Ts}$} \\
\hline & Somatic/autonomic & $44 \mathrm{Ts}$ & & \\
\hline & Total & $47 \mathrm{Ts}$ & & \\
\hline \multirow[t]{3}{*}{ Harm Avoidance } & Perfectionism & $36 \mathrm{Ts}$ & \multirow[t]{3}{*}{ MASC Total } & \multirow[t]{3}{*}{$52 \mathrm{Ts}$} \\
\hline & Anxious Coping & $48 \mathrm{Ts}$ & & \\
\hline & Total & $42 \mathrm{Ts}$ & & \\
\hline \multirow[t]{3}{*}{ Social Anxiety } & Humiliation/Rejection & $52 \mathrm{Ts}$ & \multirow[t]{3}{*}{ Anxiety Disorder Index } & \multirow[t]{3}{*}{$53 \mathrm{Ts}$} \\
\hline & Performance Fears & $58 \mathrm{Ts}$ & & \\
\hline & Total & $55 \mathrm{Ts}$ & & \\
\hline
\end{tabular}

Children's Depression Inventory (CDI)

Total score 7 points

Children's Yale-Brown Obsessive Compulsive Scale (CY-BOCS)

Obsessive points

$4 \quad$ Compulsive points

1

Total points

5/ Mild disorder

Swanson, Nolan, and Pelham-IV Questionnaire (SNAP-IV) Parents rating

$\begin{array}{lclr}\text { Inattention Scale } & 0.88 \mathrm{AP} & \text { Hyperactivity/impulsivity Scale } & 1.11 \mathrm{AP} \\ \text { Conners' Parent Rating Scale-Revised: Short Form (CPRS-R:S) } & & \\ \text { Oppositional } & 65 \mathrm{AP} & \text { Cognitive Problems/Inattention } & 56 \mathrm{AP} \\ \text { Hyperactivity } & 70 \mathrm{AP} & \text { ADHD Index } & 62 \mathrm{AP}\end{array}$

Legend: WP: Wechsler points; SP: standard points; \%P: percentile points; Ts: T-score; Zs: Z-score; C: clinical; B: borderline; ADHD: Attention deficit hyperactivity disorder; OCD: obsessive-compulsive disorder; EBD: eating behavior disorder; GAD: generalized anxiety disorder; AP: average points; *: clinical point 


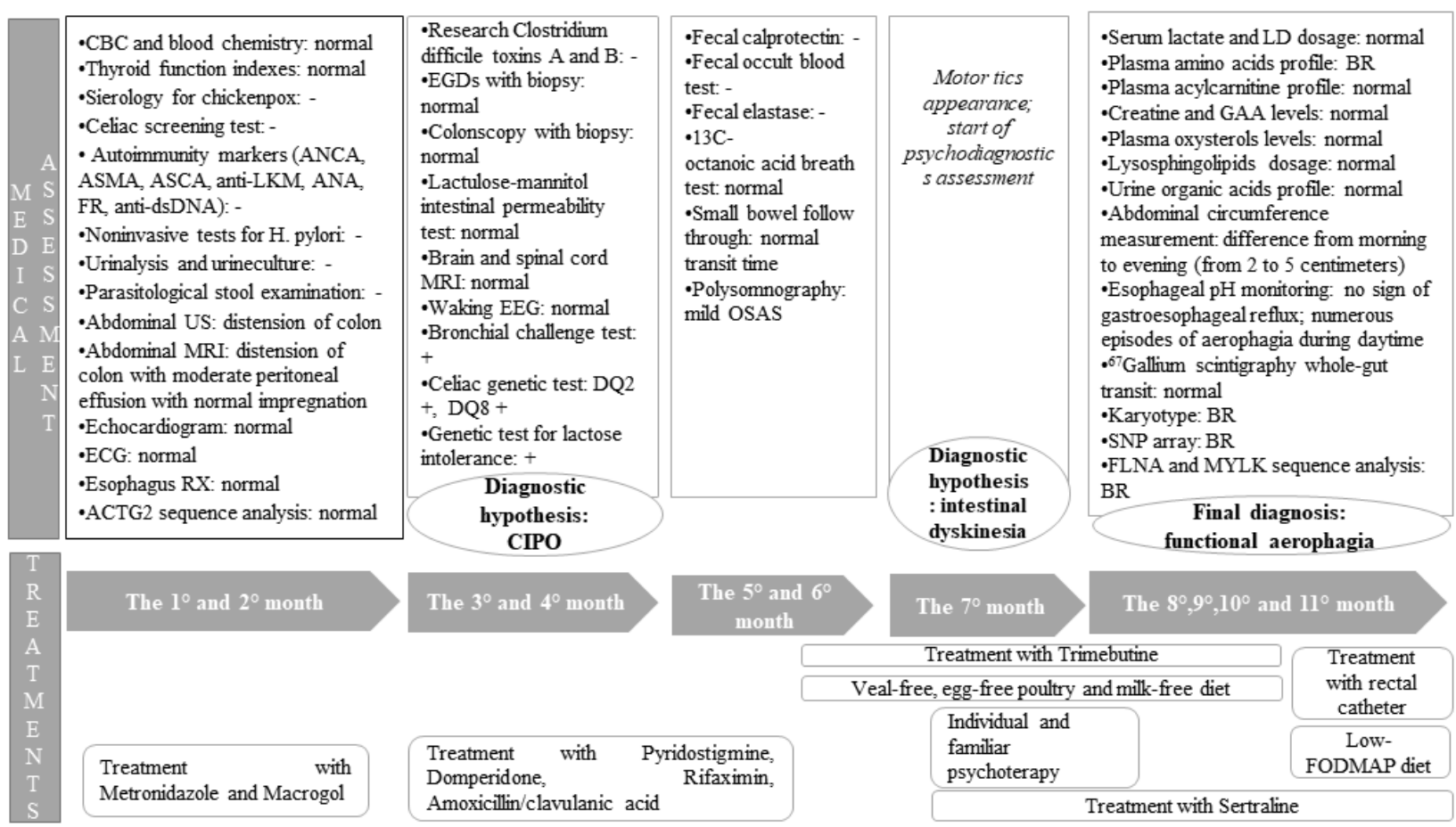

Legend: CBC: complete blood cont; US: ultrasonography; MRI: magnetic resonance imaging; ECG: electrocardiogram; RX: radiography; ACTG2: actin gamma 2; EGDs: Esophagogastroduodenoscopy; EEG: electroencephalogram; CIPO: chronic intestinal pseudo-obstruction; LD: Lactate dehydrogenase; GAA: Guanidinoacetic acid; SNP: single nucleotide polymorphism; FLNA: filamin A; MYLK: myosin light chain kinase;

FODMAF: fermentable, oligosaccharides, monosaccharides, and polyols; -: negative result; BR: being reported

In the center: the months since the onset of symptoms. In the upper part, the squares represent the hospitalizations, inside there are clinical investigations carried out; in the ovals the proposals for diagnosis; in italic style there are signs and symptoms that have appeared. On the bottom, treatments and drug therapies carried out

Figure 1. Medical assessment and treatments storyline

Moreover, some studies suggest that patients with hypermobility syndrome, including those with generalized joint hypermobility, frequently have psychiatric comorbidities, especially somatoform and mood disorders (Lam et al 2020, Wasim et al 2019).

Most of the studies seem to identify psychosocial factors involved in the pathogenesis of FD, emphasize the importance of a multidisciplinary approach on managing the case, and suggest that psychodiagnostics assessment should be performed simultaneously with other clinical examinations (Malas et al. 2017, Sitholey \& Agarwal 2008, Morabito et al. 2014). These methods allow to a fast diagnosis, avoiding inappropriate medical interventions (Malas et al 2017, Bujoreanu et al 2014, Sitholey \& Agarwal 2008, Morabito et al 2014). In this case report, the evaluation and treatment of psychological characteristics were carried out after months. The several hospitalizations and invasive procedures have caused an iatrogenic effect: over time, the patient experienced high levels of anxiety and new signs (motor tics) and regressive behavior.

Since the parents of the 8-years-old boy were very worried about unknown diagnosis, they increased the demand for interventions giving importance mainly to somatic symptoms while neglecting the emotional consequences. This type of patients have difficulty expres- sing distress through speech, they fail to develop a more functional coping for the expression of discomfort so that they are reinforced in their 'sick' role; the increase in pain manifestations is correlated with a worse outcome in these disorders (Caes et al 2017).

The presence of psychosomatic symptoms, loss of appetite and separation anxiety could indicate difficulty in expressing one's identity in this family context (Minuchin 1974). Furthermore home rectal catheters used to evacuate air might have caused enmeshment with the maternal figure: on the one hand she isn't able to contain her emotionality nor to regulate that of her son, on the other hand she is a rigid regulator of child's physiological functions. She has become "the one who gives care and relief" but who also reduced his autonomy, leading him to regression to the early stages of life. The act of therapy itself seems to symbolize a "psychic incest" and this could cause feelings of anger that the child has expressed with oppositional behavior against the father.

\section{CONCLUSIONS}

FGD are common during childhood and cause frequent consultations. The described patient's history highlights the importance of taking into consideration the frequent association with psychiatric comorbidities of 
these disorders in order to make, according with ROMA IV criteria, an early recognition that helps to reduce parental and patient anxiety and prevent unnecessary hospital admissions and medical investigations.

\section{Acknowledgements: None.}

Conflict of interest: None to declare.

\section{Contribution of individual authors:}

Consuelo Basile \& Federica Gigliotti: idea, concept and design of the article, literature search, writing manuscript.

Michela Colaiori \& Ignazio Ardizzone: writing manuscript, continuous reviewing.

All authors approved the final manuscript.

\section{References}

1. Bujoreanu $S$, Randall E, Thomson K, Ibeziako P: Characteristics of medically hospitalized pediatric patients with somatoform diagnoses. Hosp Pediatr 2014; 4:283-290

2. Caes L, Orchard A, Christie D: Connecting the MindBody Split: Understanding the Relationship between Symptoms and Emotional Well-Being in Chronic Pain and
Functional Gastrointestinal Disorders. Healthcare (Basel) 2017; 5:93

3. Cozzi G, Barbi E: Chronic school absenteeism as a diagnostic clue for paediatricians. $J$ Paediatr Child Health 2020; 56:191-193

4. Hyams JS, Di Lorenzo C, Saps M, Shulman RJ, Staiano A, van Tilburg M: Childhood functional gastrointestinal disorders: child/adolescent. Gastroenterology 2016; 150:1456-1468

5. Lam CY, Palsson OS, Whitehead WE, et al: Rome IV Functional Gastrointestinal Disorders and Health Impairment in Subjects With Hypermobility Spectrum Disorders or Hypermobile Ehlers-Danlos Syndrome. Clin Gastroenterol Hepatol, 2020

6. Malas N, Ortiz-Aguayo R, Giles L, Ibeziako P: Pediatric somatic symptom disorders. Curr Psychiatry Rep 2017; 19:11

7. Minuchin S: Families and family therapy. New York. Harvard University Press 1974

8. Morabito $G$, Romeo C, Romano C: Functional aerophagia in children: a frequent, atypical disorder. Case Rep Gastroenterol 2014; 8:123-128

9. Sitholey P, Agarwal V: Clinical practice guidelines for the management of pediatric somatoform disorders. Indian $J$ Psychiatry 2008; 157-167

10. Wasim S, Suddaby JS, Parikh M, et al: Pain and gastrointestinal dysfunction are significant associations with psychiatric disorders in patients with Ehlers-Danlos syndrome and hypermobility spectrum disorders: a retrospective study. Rheumatol Int 2019; 39:1241-1248

Correspondence:

Consuelo Basile, MD

Multispecialty Department Child Neuropsychiatry, Department of Human Neurosciences,

Sapienza University of Rome

Via dei Sabelli 108, 00185, Rome, Italy

E-mail:consuelo.basile@uniroma1.it 